\title{
Esperando un diagnóstico: experiencias de padres de niños con sospecha de discapacidad
}

\section{Waiting for a diagnosis: experiences of the parents of children with asuspected disability}

\author{
Samanta López, Silvia López-Larrosa y Manuel Peralbo \\ Universidade da Coruña
}

\begin{abstract}
Resumen
El diagnóstico de una discapacidad o trastorno del desarrollo puede tener lugar en varios momentos, y puede extenderse en el tiempo. Durante el momento pre-diagnóstico, la familia vive una etapa de frustración al no saber qué ocurre y qué hacer. Los servicios de atención temprana son un recurso al que son derivadas estas familias. Para conocer cómo viven las familias este tiempo de espera, se ha hecho un estudio con 12 padres y madres. Los resultados indican que la duración del proceso y la relación con los profesionales son fundamentales a la hora de afrontar la situación.

Palabras clave: padres, diagnóstico, sospecha, atención temprana.
\end{abstract}

\begin{abstract}
Getting a diagnosis of a disability or a developmental delay may happen in different moments and may expand in time. During the pre-diagnosis period, families experience frustration as they do not know what happens and what to do. Early attention services are a resource to families. In order to know how families live these wait- times, 12 parents were studied. Results show that the length of the wait-time and the relationships developed with the professionals are core variables to face this family situation.
\end{abstract}

Key words: parents,diagnosis, suspicion, early attention.

La meta del presente trabajo es analizar el modo en que la espera de un diagnóstico afecta a las relaciones familiares y de pareja de los padres con niños con trastornos del desarrollo o discapacidad, o que aún no tienen el diagnóstico, y que acuden a un centro de atención temprana. No se dispone en la actualidad en España de datos contrastados acerca de esta situación. Sin embargo, varias investigaciones internacionales han abordado esta cuestión, aunque todas ellas se hicieron después de que los padres tuviesen el diagnóstico de lo que le ocurría a su hijo/a (Dixon-Woods, Findlay, Young, Cox y Heney,2001; Finnvold, 2009; Graungaard y Skov, 2007; Knalf, Ayres, Gallo, Zoeller y Breitmayer, 1995; Mitchell y Holdt, 2014). La literatura nos ofrece varios elementos clave cuando se investiga este periodo, como son la duración del proceso diagnóstico o la relación padres-profesional.

El diagnóstico de una discapacidad o un trastorno del desarrollo puede establecerse en varios momentos de la vida. El diagnóstico se puede producir antes del nacimiento del niño, en el momento del nacimiento o pasado un tiempo desde el mismo. Knalf et al. (1995) también apuntan que se puede hacer inmediatamente después del comienzo de los síntomas, años después del comienzo de los síntomas o en ausencia de síntomas.

Como indican Mitchell y Holdt (2014), los padres sienten que los profesionales no quieren emitir un diagnóstico demasiado pronto, lo que provoca que la duración del período pre-diagnóstico se prolongue en el tiempo. La extensión temporal de este momento influye en la satisfacción o no de los padres. Dixon-Woods et al. (2001) y Finnvold (2009) apuntan que la satisfacción de los padres se verá incrementada por la rapidez con la que obtienen el diagnóstico de sus hijos. Además, el uso de estrategias de afrontamiento es menor cuando el diagnóstico es tardío (Graungaard y Skov, 2007), Las experiencias iniciales influyen en la adaptación al diagnóstico (Dixon-Woods et al., 2001; Graungaard y Skov, 2007).

En cuanto a la relación con el profesional, las investigaciones indican que hay mucho que mejorar por parte de los encargados del diagnóstico. Los padres afirman que no existe cooperación alguna en estos momentos (Dixon-Wood et al, 2001), tienen la necesidad de hacer algo (Graungaard y Skov, 2007) y ven cómo el profesional no les deja. También dicen que no se les escucha (Lundeby y T॰ssebro, 2008) y que tratan a los niños como casos y no como personas, viendo solo las discapacidades en vez de lo que sí pueden hacer (Graungaard y Skov, 2007).

Otro pilar fundamental de esta investigación es la atención temprana. A pesar de que la familia figura como destinataria de los servicios de atención temprana, no hay una delimitación clara de la atención que se debe ofrecer a la familia (Turnbull, Summers y Turnbull, 2007). Uno de los objetivos de la atención temprana es atender y cubrir las necesidades y demandas de la familia y del entorno en el que vive el niño (G.A.T., 2000), pero pocas son las intervenciones hechas con la familia, limitándose la mayoría de ellas a enseñarle a los padres cómo ayudar y establecer una relación con su hijo. Botana y Peralbo (2014) indican que el niño, su familia y su entorno forman parte de un sistema donde todos ellos están en constante interacción, y que el funcionamiento de cada una de las partes influye en las demás. Si la atención temprana busca ayudar al niño, debería tener más en cuenta el bienestar de los demás sistemas que influyen en su vida.

Si a la falta de programas destinados a la familia desde la atención temprana se le añade la carencia de investigaciones sobre el período pre-diagnóstico en 
España, se puede observar como hay un vacío que es necesario cubrir.

Así pues, los objetivos de este estudio se pueden resumir en:

- Analizar el tiempo de sospecha: la influencia en las relaciones familiares y en las relaciones con los profesionales.

- Determinar cómo afrontan los padres el diagnóstico de su hijo desde los servicios de atención temprana.

\section{Método}

\section{Participantes}

Los participantes en este estudio fueron 11 madres y un padre, con hijos cuyas edades oscilaban entre 1 y 6 años. Todos ellos son usuarios de un servicio de atención temprana gallego. Aunque algunos de estos niños no tienen un diagnóstico de la dificultad que presentan, hay 6 que sí que lo tienen. Hay variedad en los diagnósticos establecidos, como síndrome de Down, maullido de gato, retraso global del desarrollo, retraso en la comunicación y el lenguaje y trastorno del espectro autista. Únicamente dos familias conocen el diagnóstico desde el momento del nacimiento.

En cuanto a las características que presentan los padres, son muy variadas. La edad de la participante más joven de este estudio es de 21 años, mientras que la de mayor edad tiene 48 años. La media de edad de los padres es de 36 años. Hay 10 participantes que están casados, una madre soltera y una madre divorciada. En tres familias en el hogar conviven los abuelos del niño con dificultades. Diez familias tienen más hijos, y cuatro tienen otro niño o niña con problemas, como falta de atención (dos casos), neuropatía auditiva profunda y síndrome de Asperger.

\section{Materiales}

La recogida de datos se produjo en dos momentos y mediante dos técnicas diferentes. En primer lugar se realizó un grupo de discusión con tres madres. Las preguntas elicitadoras de la discusión en el grupo giraron en torno a los siguientes temas: el diagnóstico, la sospecha de alguna dificultad y el período pre-diagnóstico y la relación con los profesionales. Con la información recogida, se elaboró un cuestionario compuesto por 52 preguntas, divididas en tres apartados: datos sociodemográficos sobre la familia, el diagnóstico (beneficios e inconvenientes de estar diagnosticados, cómo éste ha influido en la familia y en las relaciones familiares, quién les ha dado el diagnóstico, etc.) y la sospecha.

\section{Procedimiento}

Para poder contactar con los participantes, se solicitó un permiso a la mancomunidad a la que pertenece el servicio de atención temprana. Una vez obtenido el permiso, se contactó con una profesional del centro de atención temprana y ella habló con los padres y madres para constituir el grupo de discusión. Debido a la incompatibilidad de horarios de los padres y madres, únicamente tres madres pudieron participar en esta actividad. El grupo de discusión se realizó en el propio servicio de atención temprana. Fue dirigido por dos profesionales ajenas al centro. Tras la realización del mismo, se elaboró un cuestionario partiendo de los comentarios extraídos del grupo de discusión. Dicho cuestionario fue entregado a los padres y madres participantes por las trabajadoras del servicio de atención temprana. Una vez cubierto, se lo devolvían a las trabajadoras, que a su vez se lo remitían a los responsables de esta investigación.

\section{Resultados}

\section{La sospecha}

En cuanto a la duración del momento de la sospecha, hay tres padres que coinciden en que su espera fue de 6 meses. Sin embargo, otros padres indican que obtuvieron el diagnóstico en menos tiempo, concretamente en 1 mes, mientras que otros superan con creces esta duración, llegando al año, a los dos años e incluso a los 4 años.

Este período se describe en general como negativo, de dolor y sufrimiento, intranquilidad, angustia o desesperación, donde los padres piensan cómo será el futuro y cómo ayudar al niño. Por otro lado, hay una pequeña parte de los padres que indican que este período ha sido rápido y que se han sentido tranquilos.

En cuanto a las relaciones familiares, la relación padre/madre-hijo/a es generalmente buena, así como la relación de pareja, la relación con los otros hijos y la relación con otras personas, aunque hay excepciones. La relación entre hermanos sigue la misma dinámica que las anteriores, por lo general no presenta problemas, pero sí es cierto que indican que hay alguna que es dura o difícil.

Lo que ayudó a las familias durante este tiempo ha sido principalmente el apoyo profesional, la familia y el centro de atención temprana. Por otra parte, el abanico de lo que no les ayudó es amplio, como no tener el diagnóstico, tener el alta y seguir con el problema, la negación de pruebas y terapias, la negación de ayudas profesionales, ver cómo el niño no avanza o los prejuicios sobre la etiqueta.

Sobre la relación con los profesionales, la mayor parte de los padres consideran que sí que hicieron algo útil durante este tiempo las trabajadoras de atención temprana, las psicopedagogas, neuropediatras o pediatras, que les han hecho más pruebas, les aportaron calma y trabajaron con el niño. Por lo general no tienen queja alguna de los profesionales, excepto tres madres que revelan que los profesionales no les dieron importancia a las dificultades del niño, no les derivaron al servicio necesario o no les ayudaron. Gran parte de los profesionales encargados del diagnóstico han preguntado a los padres su opinión o sus conocimientos sobre el niño/a, aunque algunos indican que no los tienen en cuenta.

Únicamente dos madres consideran que el tiempo de espera ha influido en la forma de recibir y aceptar el diagnóstico de su hijo. Otros cinco padres consideran que no les ha influido, porque aceptan el diagnóstico sin problema, lo intuyen y lo van asimilando. 
En cuanto a qué han echado en falta durante la sospecha, los padres indican amabilidad y tacto, más rapidez, ayuda psicológica a los padres, detenerse con el paciente, empezar antes el tratamiento o explicaciones de un profesional de los posibles diagnósticos y que les aporten tranquilidad. Los padres piden comprensión y paciencia, apoyo a las familias, pensar más en los niños, suavidad al transmitir el diagnóstico, más información sobre qué hacer o a dónde acudir y tranquilidad.

Más de tres cuartas partes de los padres consideran que debería existir un servicio especializado para ayudar a los padres durante este momento de sospecha, para indicarles qué hacer con el niño, ayudar personalmente a cada uno, ayudarles en las relaciones de pareja, ayudar a los hermanos e incluso, a la familia cercana, el colegio o la guardería.

\section{El diagnóstico}

Únicamente la mitad de los hijos de estos padres tienen un diagnóstico confirmado por algún profesional. El camino recorrido para conseguir el diagnóstico ha sido complicado, ya que 7 de los 12 participantes apunta que ha tenido que acudir a varios servicios para obtenerlo, visitar a distintos especialistas como neurólogos o neuropediatras, logopedas, pediatras o médicos rehabilitadores. Éstos fueron los que se encargaron de establecer el diagnóstico en gran parte de los casos, y en otra buena parte lo hicieron en colaboración con los servicios de atención temprana.

En cuanto al momento de recibir la noticia, los participantes apuntan que en todos los casos estaba al menos uno de los dos progenitores. En dos casos el diagnóstico se lo dieron a la madre sola, en tres a ambos padres y en otro caso a ambos padres y a otro familiar que les acompañaba. A la hora de trasmitir la noticia, los profesionales encargados aportaron informes médicos en gran parte de los casos. En cuanto a la información que les daban además de la “etiqueta”, los profesionales se centraron en primer lugar en posibles terapias, luego en la explicación de la dificultad, pautas para relacionarse y enseñar al niño, a qué recursos acudir y qué ayudas pueden solicitar. Otra información como la posible evolución de la dificultad o asociaciones relacionadas con el diagnóstico la dieron con menos frecuencia. Cinco padres afirmaron que el profesional que les dio el diagnóstico se puso en contacto con ellos pasado un tiempo o les ofreció la posibilidad de acudir a él en el caso de que les surgiesen dudas. Los padres tienen diversas opiniones sobre lo que hicieron bien y lo que sería mejorable del profesional en el momento de recibir el diagnóstico. Algunos padres afirman que el profesional encargado del diagnóstico lo hizo todo bien, otros que lo que hizo bien fue dar información, u otras tareas como hacer más pruebas para descartar otros trastornos, tratar el tema con normalidad, dar una explicación sencilla, dar esperanzas o empezar a tratar el problema. En cuanto a lo que sería mejorable, en lo que coinciden algunos padres es en el tiempo de espera. Además, consideran que tendría que cambiar la actitud fría e insensible del profesional o disponer de más servicios en la Seguridad Social. La respuesta del único padre participante a esta pregunta es que lo que debería mejorar es que el diagnóstico no fuese el que ha recibido.

Una vez conocido el diagnóstico, 4 de los 6 padres con hijos con diagnóstico afirmaron sentir tranquilidad. También indicaban que, a diferencia del anterior periodo, donde no sabían aún lo que ocurría con su niño o niña, ahora sabían cómo ayudarle, considerado éste el mayor beneficio de tener la "etiqueta”. El tener el diagnóstico también les ayuda a asimilar mejor lo que les espera en el futuro, a afrontar el problema o a explicarles a los demás qué es lo que ocurre con su hijo. Algo que los padres consideran importante de tener el diagnóstico "formal" es la facilidad que ello supone a la hora de solicitar ayudas o becas, ya que sin el diagnóstico tienen que contar con la voluntad de los funcionarios o profesionales para conseguir algo para el niño, como indicó una de las madres. En cuanto a los aspectos negativos de tener el diagnóstico, parte de los padres afirma que no hay ninguno, mientras que otros inciden en el trato diferente que le pueden dar al niño o niña. Es destacable la respuesta de una de las madres, que indica que un aspecto negativo del diagnóstico es el momento de recibir la noticia, aunque después también se asimila mejor lo que le pasa al niño.

En cuanto a la influencia del diagnóstico en la familia, varios participantes indicaron que el diagnóstico no ha influido en los hermanos de forma alguna, aunque hay otros que dicen que los niños ven que su hermano no es igual o ponen límites en los juegos y actividades. En cuanto a la influencia en otros familiares, se pueden ver las dos caras de la moneda, es decir, hay familiares en los que el diagnóstico no ha tenido ningún efecto, mientras que a otros les cuesta aceptarlo y comparan al niño con los demás.

En lo tocante a las relaciones familiares una vez se cuenta con un diagnóstico, hay padres que indican que la relación matrimonial no se ha visto afectada, aunque hay otros que indican que es duro y que se viven momentos de frustración por el futuro. También se ha señalado el apoyo mutuo y la unión de la pareja en este momento, y que ambos padres lo que quieren es lo mejor para los hijos. En cuanto a la relación con el niño, el diagnóstico ha ayudado a los padres a saber cómo ayudarle y cómo tratarlo y relacionarse con él. La relación con los otros hijos o bien se mantiene igual, sin haber diferencias entre ambos, o bien se pasa a estar pendientes de cada actividad para evaluar al niño sin problemas por si hay algo raro en su conducta. Es significativa la respuesta de una de las madres, que indica que lo que le enseñan los profesionales para trabajar con el niño con diagnóstico lo puede aplicar al otro hijo.

\section{Discusión}

El camino al diagnóstico es una etapa de incertidumbre en la vida familiar, tanto para los padres como para los hijos. Antes de llegar al diagnóstico, la familia realiza un recorrido por multitud de profesionales y especialistas, lo que provoca que el camino se haga más y más largo. Desde que los padres acuden con el niño al primer profesional, generalmente el pediatra de atención primaria, hasta que les derivan a neuropediatras, 
logopedas, médicos rehabilitadores, etc., pueden pasar varios meses, como indican los participantes. Según Finnvold (2009), el vagar de un profesional a otro retrasa el diagnóstico.

Los datos de esta investigación sobre la relación de los padres con el profesional durante la sospecha muestran que la mayoría de los padres afirman que los profesionales sí les han tenido en cuenta, les han preguntado su opinión, e incluso les han aportado calma en el momento previo al diagnóstico. Sin embargo, hay otros que echan en falta un trato con más tacto y más rapidez, lo cual puede coincidir con las experiencias de padres de otros lugares del mundo (Lundeby y Tøssebro, 2008).

El momento pre-diagnóstico, descrito negativamente por parte de los padres, es un periodo en el que los padres pueden sentir estrés y soledad (Mitchell y Holdt, 2014). El recibir el diagnóstico, pese al resultado, aporta a los padres tranquilidad: ahora saben qué es lo que le pasa a su hijo y, por lo tanto, pueden comprender su comportamiento y explicárselo así a los demás (Mitchell y Holdt, 2014). Ahora pueden recuperar el control de su vida y afrontar la nueva situación (Graungaard y Skov, 2007).

Cuando se da el diagnóstico, el contenido del mismo varía. Además de la "etiqueta”, los profesionales suelen aportar otra información como posibles terapias, más información sobre la discapacidad o trastorno del niño, indicaciones para los padres sobre cómo relacionarse con su hijo y posibles ayudas, tanto profesionales como económicas. Según la investigación realizada por la G.A.T. (2013), de los anteriores contenidos el único que coincide con sus datos es la mayor información sobre el diagnóstico. Los datos de esa investigación indican que los profesionales no suelen dar un mensaje con gran variedad de contenidos en el momento del diagnóstico.

Los resultados del presente estudio muestran que los participantes no sienten que las relaciones familiares se vean afectadas ni antes de conocer el diagnóstico, ni después, aunque hay una pequeña parte de los padres con diagnóstico que sí indican que es duro y que hay preocupación por el futuro. Por otro lado, la relación con el hijo que presenta alguna dificultad parece verse mejorada después del diagnóstico, ahora saben lo que pueden hacer para ayudarle y relacionarse adecuadamente con él.

Para afrontar la presencia de una dificultad, los padres consideran que el apoyo profesional, la familia y el centro de atención temprana son fundamentales. Obtener el diagnóstico puede brindar ese apoyo (Mitchell y Holdt, 2014).

\section{Referencias}

Botana, I. \& Peralbo, M. (2014). Familia, estrés y atención temprana. Revista de Estudios e Investigación en Psicología y Educación, 1 (1), 55-63. http://dx.doi.org/10.17979/2386-7418.reipe.20 $14 \mathrm{v} 1 \mathrm{i} 1.23 .55$

Dixon-Woods, M., Findlay, M., Young, B., Cox, H. \& Heney, D. (2001). Parents' accounts of obtaining a diagnosis of childhood cancer. The Lancet, 357, 670-674.

http://dx.doi.org/10.1016/S0140-6736(00)04130-1

Federación Estatal de Asociaciones de Profesionales de la Atención Temprana (G.A.T.) (2013). La primera noticia. Estudio sobre los procedimientos profesionales, las vivencias y las necesidades de los padres cuando se les informa de que su hijo tiene una discapacidad o un trastorno del desarrollo. Madrid: Real Patronato sobre Discapacidad.

Finnvold, J. E. (2009). Childhood asthma: Regional and social inequalities in parents' perceptions of the diagnostic process in Norway. Norsk Geografisk Tidsskrift- Norwegian Journal of Geography, 63, 166-174.

http://dx.doi.org/10.1080/00291950903238982

Graungaard, A. H., \& Skov, L. (2007). Why do we need a diagnosis? A qualitative study of parents' experiences, coping and needs, when the newborn child is severely disabled. Child: Care, Health and Development, 33(3), 296-307. http://dx.doi.org/10.1111/j.1365-2214.2006.00666.x

Grupo de Atención Temprana (G.A.T.) (2000). Libro blanco de la Atención Temprana. Madrid: Real Patronato de Prevención y de Atención a Personas con Minusvalía.

Knafl, K., Ayres, L., Gallo, A., Zoeller, H. \& Breitmayer, B. (1995). Learning from stories: parents' accounts of the pathway to diagnosis. Pediatric Nursing, 21 (5), 411-415.

Lundeby, H. \& $\mathrm{T} \oslash$ ssebro, J. (2008). Exploring the experiences of "not being listened to" from the perspective of parents with disabled children. Scandinavian Journal of Disability Research, 10 (4), 258-274.

http://dx.doi.org/10.1080/15017410802469700

Mitchell, C., \& Holdt, N. (2014). The search for a timely diagnosis: Parents' experiences of their child being diagnosed with an autistic spectrum disorder. Journal of Child and Adolescent Mental Health, 26(1), 49-62. http://dx.doi.org/10.2989/17280583.2013.849606

Turnbull, A. P., Summers, J. A. \& Turnbull, R. (2007). Family supports and services in early intervention: a bold vision. Journal of Early Intervention, 29 (3), 187-206.

http://dx.doi.org/10.1177/105381510702900301

\section{Agradecimientos}

Se agradece a los padres y madres participantes en esta investigación su participación, y también a las trabajadoras del servicio de atención temprana su colaboración. 